\title{
CARACTERES EPIDÉRMICOS FOLIARES DE PLANTAS ORNAMENTALES, TÓXICAS PARA ANIMALES DOMÉSTICOS.
}

\section{LEAF EPIDERMAL CHARACTERS OF ORNAMENTAL PLANTS, TOXIC FOR DOMESTIC ANIMALS.}

\author{
Patricia Nasca de Zamora ${ }^{a}$
}

\section{RESUMEN}

El tejido epidérmico de los vegetales presenta un diseño característico para cada especie, de manera que muchas plantas pueden reconocerse a través de su estudio microscópico. Además, este tejido resiste el pasaje por el tracto digestivo de los animales, permaneciendo prácticamente inalterable. Basados en este hecho, los investigadores Baungartder y Martin (1939) idearon la técnica microhistológica, que consiste en estudiar la composición botánica de la dieta de animales herbívoros a través del análisis microscópico de fragmentos de epidermis vegetales encontrados en la materia fecal de los mismos. A lo largo de los años, el uso de esta técnica se extendió ampliamente, adaptándose a diferentes objetivos, tanto ecológicos como económicos.

El objetivo del presente trabajo, que se llevó a cabo en la provincia de Tucumán, República Argentina, es describir las epidermis foliares de cuatro especies ornamentales tóxicas, a fin de aportar datos para la confección de una clave dicotómica de identificación de las mismas, para ser usada como elemento de diagnóstico en medicina veterinaria.

Se extrajeron en laboratorio - con diversas técnicas - epidermis foliares de Evonymus japonicus, Hedera helix, Ficus benjamina y Vinca difformis; especies ornamentales citadas como tóxicas en la bibliografía veterinaria y cultivadas habitualmente en jardines particulares y espacios públicos de la Provincia. Se confeccionaron preparados microscópicos permanentes, se tomaron registros fotográficos y se describieron las características epidérmicas de cada especie, teniendo en cuenta la forma de las células, la presencia o ausencia de estomas, presencia de tricomas, tipos de estomas, tipos de tricomas, etc.

Hedera helix y Vinca difformis presentan células epidérmicas de contorno lobulado, mientras que las células epidérmicas de Evonymus japonicus. y Ficus benjamina son isodiamétricas y poligonales. Las cuatro especies descriptas presentan estomas sólo en la zona internerval de la cara abaxial de sus hojas. Se encontraron tricomas simples solamente en la zona nerval de la epidermis adaxial de Vinca difformis, las demás epidermis son completamente glabras. Los caracteres encontrados en las especies estudiadas y volcados en las descripciones, constituyen un aporte de utilidad para la confección de una clave dicotómica de indentificación de estas plantas tóxicas a través de sus epidermis.

Palabras clave: epidermis foliar, plantas ornamentales, tóxicas, medicina veterinaria

\section{ABSTRACT}

The epidermal tissue of the vegetables presents a typical design for every species so that many plants can be recognized across its microscopic study. In addition, it resists the passage for the digestive tract of the animals, remaining practically inalterable. Based on this fact, Baungartder and Martin (1939) designed the microhistological technique, which consists of studying botanical composition of diet of herbivorous animals across the microscopic analysis of plant's epidermis fragments found in the fecal matter. Throughout the years, the use of this technique spread widely, adapting to different aims, both ecological and economic.

The aim of the present work, realized in Tucumán's province, Argentina, is to characterize epidermis of ornamental, toxic plants for domestic animals, in order to be used as element of diagnosis in veterinary medicine.

Epidermis of Evonymus japonicus, Hedera helix, Ficus benjamina and Vinca difformis were extracted in laboratory. All of them are ornamental species, mentioned like toxic in the bibliography veterinary and cultivated habitually in particular gardens and public spaces of the Province. They were made prepared microscopic permanent, photographic records took and there were described the epidermal characteristics of every species, bearing in mind the form of the cells, the presence or absence of stomata and trichomes, and types of stomata and trichomes.

Hedera helix and Vinca difformis present lobulated epidermal cells, whereas Evonymus japonicus and Ficus benjamina ones are isodiametrics and polygonal. Four species present stomata only in the internerval zone of abaxial epidermis. They were trichomes only in the nerval zone of adaxial epidermis of Vinca difformis. The epidermal characters found in the studied species, constitute an usefull contribution for the confection of a dichotomous indentification key of these toxic plants.

Key words: epidermis, ornamental toxic plants, veterinary medicine 
INTRODUCCIÓN usó para investigar la composición botánica de la dieta de

pequeños mamíferos silvestres, de venados, camélidos

A lo largo de los años, el uso de esta técnica se extendió de la Puna argentina y de la Puna chilena, etc. [4] [5] [6] ampliamente, adaptándose a diferentes objetivos. Así, se [7]. Se aplicó también en el estudio de la dieta del ganado

a Ingeniera Agrónoma, Profesora Adjunta de la Cátedra de Botánica General, Facultad de Agronomía y Zootecnia, Universidad Nacional de Tucumán, Argentina.

en una explotación caprina de la Provincia de Santiago del Estero, Argentina; [8] [9] y se usa actualmente en el reconocimiento de plantas silvestres tóxicas para el ganado [3].

No se encontraron antecedentes del uso de la técnica microhistológica en animales carnívoros u omnívoros. Sin embargo, es frecuente encontrar restos vegetales intactos en la materia fecal de animales de compañía, especialmente cachorros caninos y felinos. Estos restos vegetales provienen de la alimentación doméstica que se les proporcionan y también de plantas que estos animales mastican e ingieren por su natural curiosidad o hábitos de juego.

Existen muchas plantas ornamentales cultivadas en jardines particulares, plazas y parques que pueden producir intoxicaciones en estos animales [10]. En la mayoría de los casos estos procesos no son diagnosticados correctamente por el médico veterinario porque producen síntomas inespecíficos - tales como dermatitis o trastornos gastrointestinales - y porque generalmente el profesional carece de información adicional por parte de los propietarios del animal [11].

En el marco del proyecto de investigación denominado "Reconocimiento de plantas ornamentales, tóxicas para pequeños animales, a través de técnicas microhistológicas" que se lleva a cabo en la provincia de Tucumán, República Argentina, se elabora una colección de referencia, de preparados microscópicos y fotomicrografías de epidermis foliares de plantas comúnmente cultivadas como ornamentales, que son potencialmente tóxicas para los animales domésticos.

Esta colección está destinada a utilizarse en medicina veterinaria, como patrón de comparación, en el análisis microscópico de fragmentos de epidermis extraídos de la materia fecal de animales domésticos, presuntamente intoxicados con plantas ornamentales.

El presente trabajo tiene como objetivo describir las epidermis foliares de cuatro especies ornamentales y tóxicas a fin de aportar datos para la confección de una clave dicotómica para la identificación de las mismas.

MATERIAL Y MÉTODO
Se trabajó con hojas de Evonymus japonicus (Fam. Celastraceas), Hedera helix (Fam. Araliáceas), Ficus benjamina (Fam. Moráceas) y Vinca difformis (Fam. Apocináceas).

Se realizó la determinación taxonómica de las plantas y se recolectaron muestras de hojas completamente desarrolladas, que fueron almacenadas en frascos herméticos con fijador FAA.

La extracción de las epidermis se realizó según el método de Schultze [12], tratando las muestras con ácido nítrico concentrado y cristales de clorato de potasio. En aquellas especies cuyas epidermis no se desprendieron con facilidad, se procedió a diafanizar las hojas, según la técnica de Dizeo de Strittmater [13].

Las observaciones se realizaron con microscopio binocular marca Carl Zeitz, a 100 y 400 aumentos. Se tomaron fotomicrografías con una cámara digital marca Olympus, de 6 mega píxeles, de uso familiar.

El estudio epidérmico se hizo sobre un mínimo de diez repeticiones, describiéndose las caras adaxial y abaxial de las hojas y, en ambos casos, las zonas nerval e internerval.

Para las descripciones se tomó en cuenta la forma y proporción de las células epidérmicas propiamente dichas; la presencia, ubicación y tipo de estomas, según la clasificación de Van Cotthem [14], la presencia, ubicación y tipo de tricomas [15] y toda característica que permita su identificación y posterior diferenciación de otras especies vegetales.

\section{RESULTADOS}

I. Descripción de las epidermis de Evonymus japonicus.

\section{Epidermis adaxial}

\section{a. Zona internerval}

Células isodiamétricas, de contorno poligonal. Polígonos irregulares de 4 a 7 lados, más frecuentemente 6 , distribuidas sin un orden aparente. Ausencia de estomas. (Figura 1)

\section{b. Zona nerval}


Células semejantes a las de la zona internerval, pero ordenadas en hileras onduladas a lo largo de la nervadura. Se observan grupos de células triangulares o trapeciales que producen la formación de la onda en la hilera.

\section{Epidermis abaxial}

\section{a. Zona internerval}

Presencia de abundantes estomas. Entre ellos, células epidérmicas alargadas (Proporción 2:1) o isodiamétricas (Proporción 1:1), ambas de contorno poligonal. Polígonos irregulares de 4 a 7 lados, más frecuentemente 5 ó 6 .

Estomas anomocíticos. (Figura 2 y Figura 3)

\section{b. Zona nerval}

Ausencia de estomas. Células alargadas (Proporción 2:1 a 4:1; la primera, más frecuente), de contorno poligonal, de 4 a 6 lados, más frecuente 4 ó 5 . Alineadas en el sentido de la nervadura, en líneas rectas. Se observa una zona de transición entre las formas celulares de la zona internerval y las formas celulares sobre las venas. En esta zona las células son isodiamétricas, de contorno

poligonal, de 4 a 5 lados. (Figura 4)

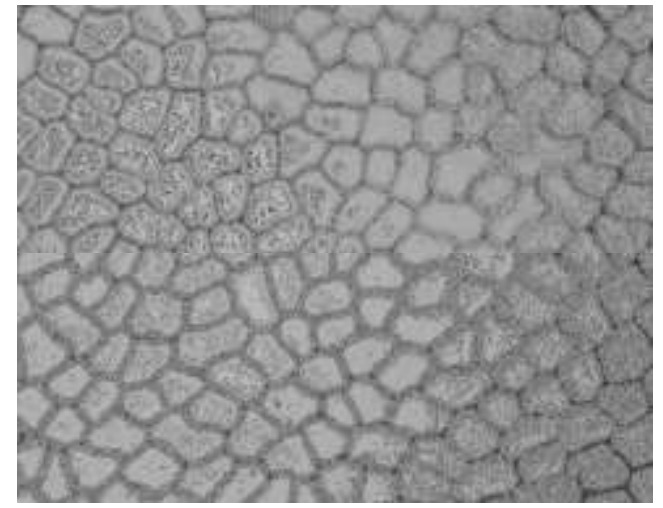

Figura 1: Epidermis adaxial de la hoja de Evonymus japonicus, zona internerval. $100 \mathrm{X}$.

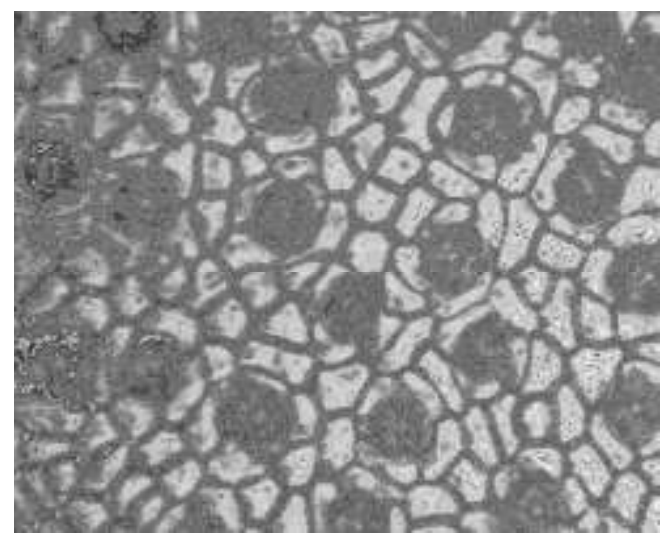

Figura 2: Epidermis abaxial de la hoja de Evonymus japonicus, zona internerval. $100 \mathrm{X}$.

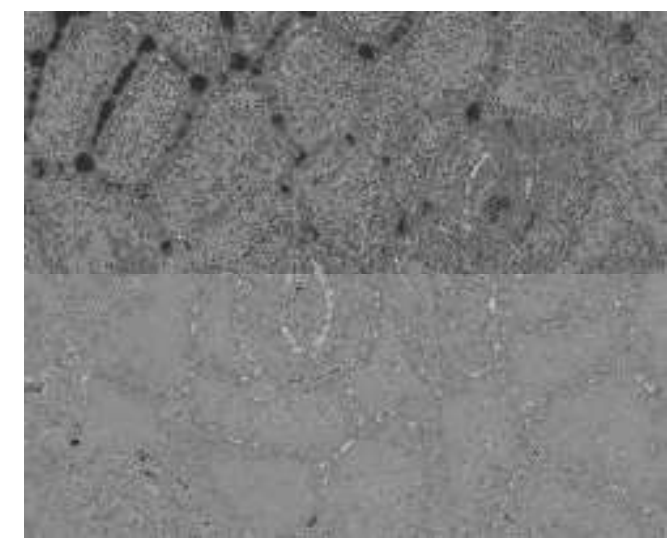

Figura 3: Epidermis abaxial de la hoja de Evonymus japonicus. Detalle de un estoma. $400 \mathrm{X}$.

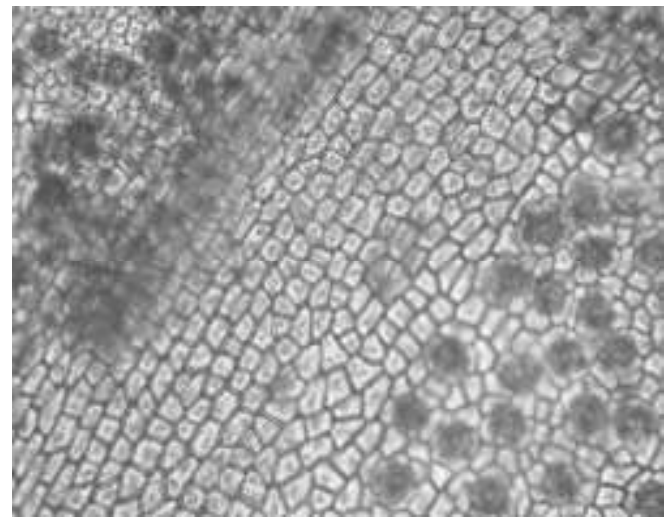

Figura 4: Epidermis abaxial de la hoja de Evonymus japonicus, zona nerval, zona internerval y zona de transición . $100 \mathrm{X}$.

II. Descripción de las epidermis de Hedera helix

1. Epidermis adaxial

\section{a. Zona internerval}

Estomas ausentes. Células epidérmicas alargadas o isodiamétricas, de contorno lobulado, con 5 a 8 lóbulos, generalmente 6. (Figura 5)

\section{b. Sobre las venas}

Células de contorno poligonal de 3 a 4 lados, isodiamétricas o ligeramente alargadas, ordenadas en filas longitudinales, paralelas a la nervadura. Se observan formas de transición entre las células lobuladas de la zona internerval y las células poligonales de la zona nerval. Ausencia de estomas. (Figura 6)

\section{Epidermis abaxial}

\section{a. Zona internerval}

Presencia de abundantes estomas. Entre ellos, células epidérmicas alargadas o isodiamétricas, de contorno lobulado, con 5 a 8 lóbulos. (Figura 7)

b. Sobre las venas 
Células de contorno poligonal, mayormente polígonos de 4 lados, isodiamétricas a ligeramente alargadas, ordenadas en filas longitudinales, paralelas a la nervadura. Paredes transversales perpendiculares $u$ oblicuas. Ausencia de estomas. (Figura 8)

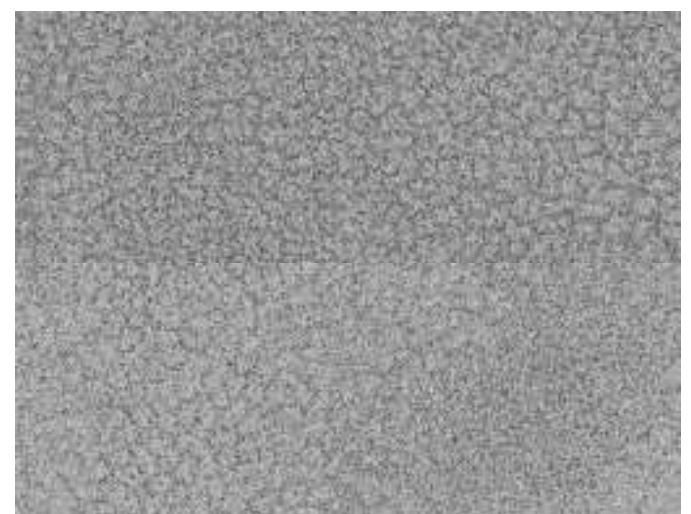

Figura 5: Epidermis adaxial de la hoja de Hedera helix, zona internerval. $100 \mathrm{X}$.

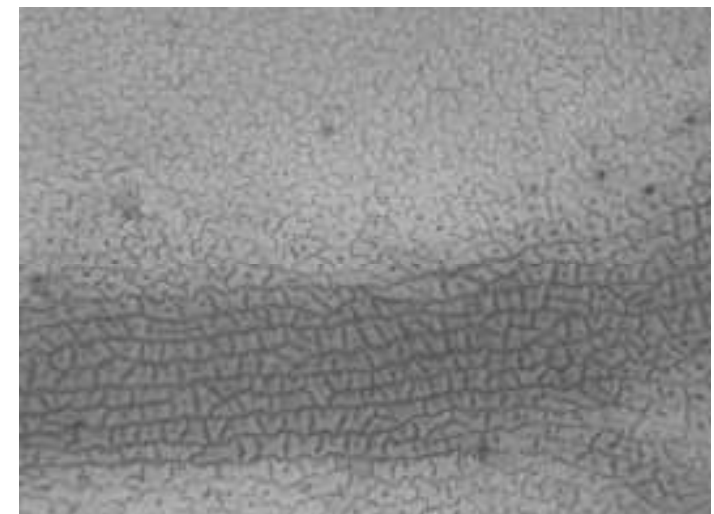

Figura 6: Epidermis adaxial de la hoja de Hedera helix, zona nerval. 100 $\mathrm{X}$.

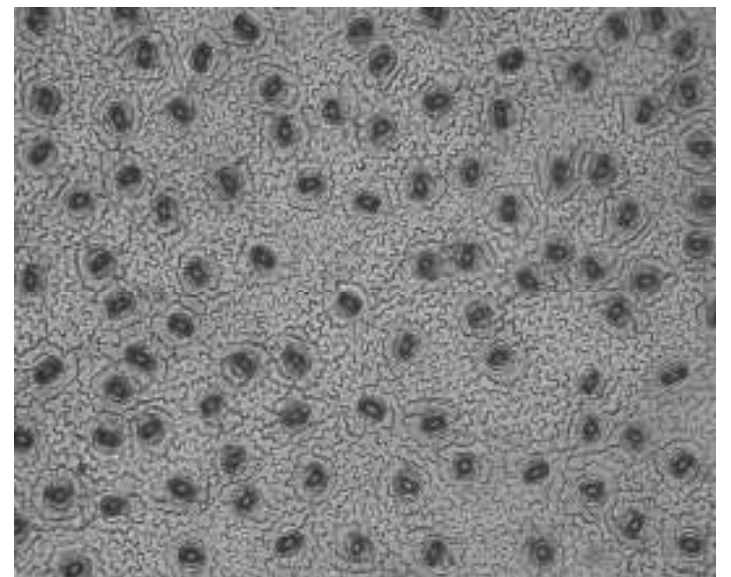

Figura 7: Epidermis abaxial de la hoja de Hedera helix, zona internerval. $100 \mathrm{X}$

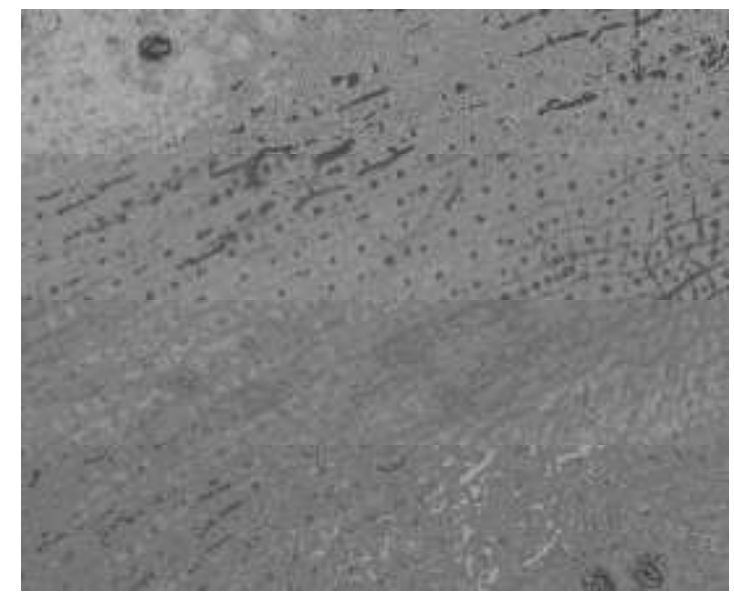

Figura 8: Epidermis abaxial de la hoja de Hedera helix, zona nerval. 100 $\mathrm{X}$

III. Descripción de las epidermis Vinca difformis.

1. Epidermis adaxial

\section{a. Zona internerval}

Estomas ausentes. Células epidérmicas alargadas 0 isodiamétricas, de contorno lobulado, distribuidas sin un orden aparente. (Figura 9)

\section{b. Sobre las venas}

Células poligonales de 4 a 6 lados, alargadas, con las paredes terminales oblicuas; ordenadas en hileras longitudinales, paralelas a la nervadura. Presencia de tricomas simples, unicelulares, abundantes sobre la vena principal y en forma aislada sobre las venas menores.

(Figuras 9 y 10)

\section{Epidermis abaxial}

\section{a. Zona internerval}

Presencia de abundantes estomas, de tamaño pequeño en relación a las células epidérmicas propiamente dichas. Células epidérmicas de contorno lobulado, semejantes a las de la epidermis adaxial. (Figura 11)

b. Sobre las venas

Células de paredes rectas, formando polígonos de 4 a 5 lados, alargadas en el sentido de la nervadura (proporción $2: 1$ y $3: 1$ ). Ausencia de estomas y tricomas. (Figura 12) 


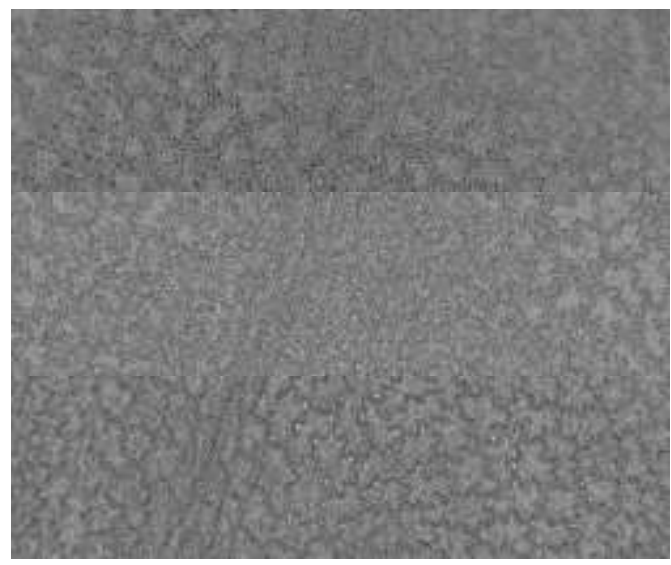

Figura 9: Epidermis adaxial de la hoja de Vinca difformis, zonas nerval e internerval. $100 \mathrm{X}$

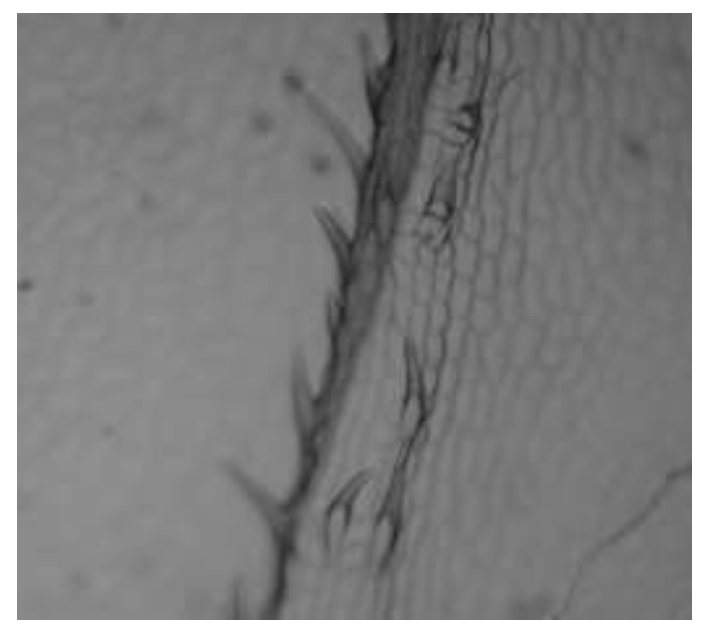

Figura 10: Epidermis adaxial de la hoja de Vinca difformis. Detalle de los tricomas sobre la nervadura principal. $100 \mathrm{X}$

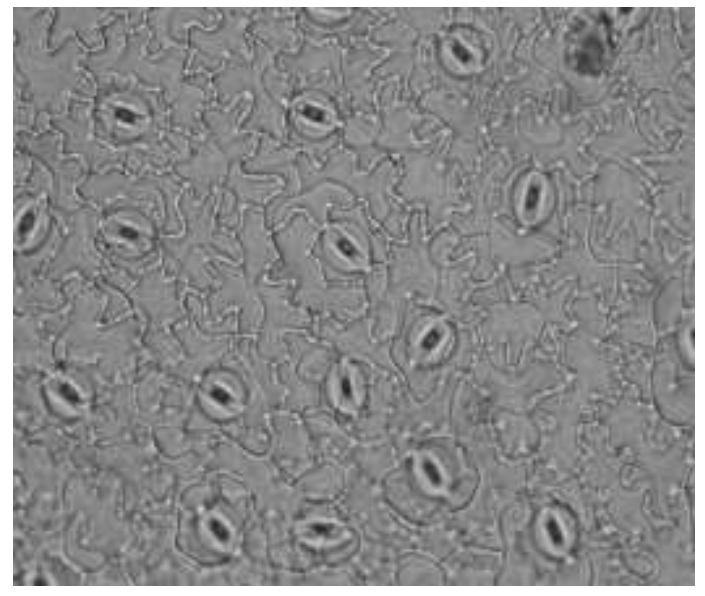

Figura 11: Epidermis abaxial de la hoja de Vinca difformis. Hoja diafanizada. $100 \mathrm{X}$

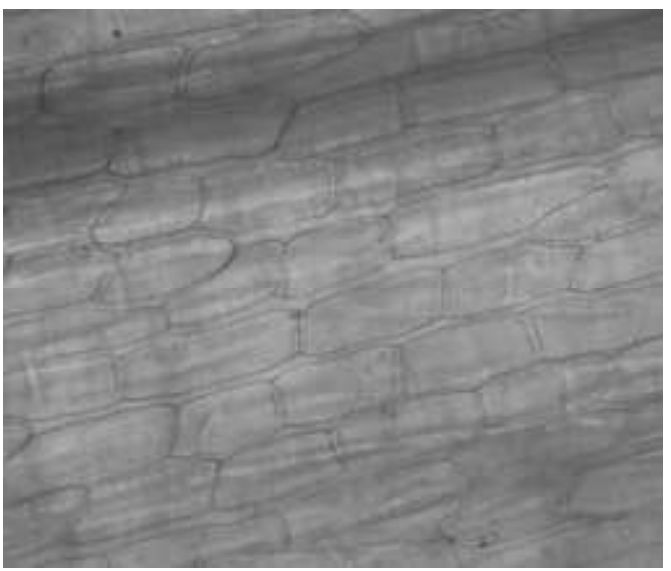

Figura 12: Epidermis abaxial de la hoja de Vinca difformis. Sobre la zona nerval. $400 \mathrm{X}$

\section{Descripción de las epidermis de Ficus benjamina}

\section{Epidermis adaxial}

\section{a. Zona internerval}

Células epidérmicas isodiamétricas, poligonales (Polígonos de 4 a 5 lados). Ausencia de estomas. Aisladamente se presentan grupos de células triangulares o subtriangulares dispuestas en forma radial, que le confieren un diseño característico. (Figura 13 y Figura 14)

\section{b. Zona nerval}

Células epidérmicas isodiamétricas, poligonales, alineadas sobre las nervaduras.

\section{Epidermis abaxial}

\section{a. Zona internerval}

Células epidérmicas poligonales, isodiamétricas, semejantes a las de la epidermis adaxial. Presencia de abundantes estomas de tipo pericíticos. (Figura 15)

\section{b. Zona nerval}

Células poligonales, isodiamétricas o alargadas, alineadas sobre la nervadura. Paredes transversales generalmente perpendiculares, a veces oblicuas.

Ausencia de estomas. (Figura 15)

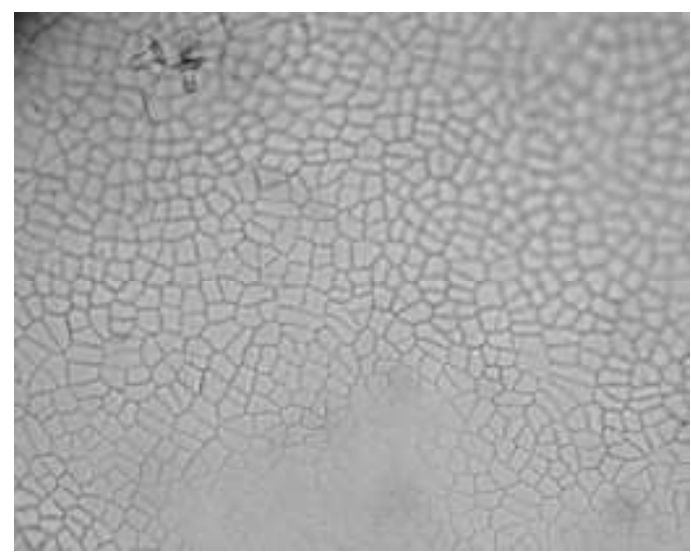


Figura 13: Epidermis adaxial de la hoja de Ficus benjamina, zona internerval. $100 \mathrm{X}$

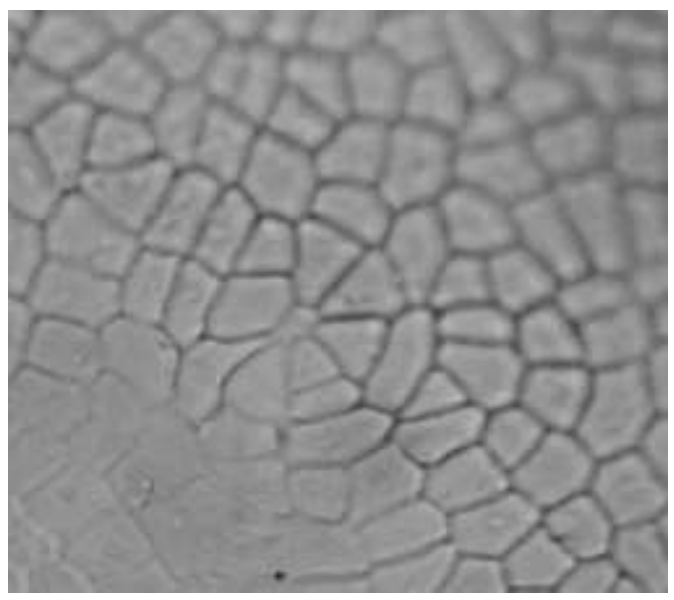

Figura 14: Epidermis adaxial de la hoja de Ficus benjamina. Detalle de un grupo de células triangulares, dispuestas en forma radial. $400 \mathrm{X}$

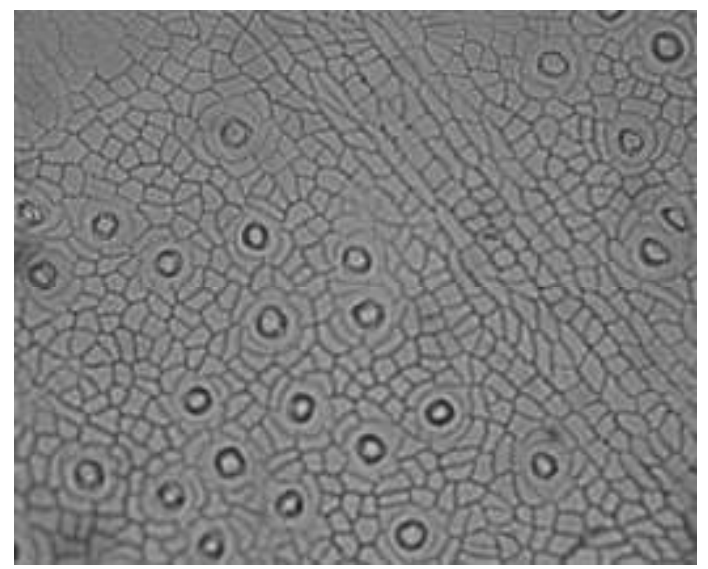

Los preparados microscópicos y fotomicrografías fueron incorporados a la colección histológica de referencia que se elabora en el marco del proyecto de investigación denominado "Reconocimiento de plantas ornamentales, tóxicas para pequeños animales, a través de técnicas microhistológicas".

Se encuentran depositados en la histoteca del Laboratorio de Histología Vegetal de la cátedra de Botánica General de la Facultad de Agronomía y Zootecnia de la Universidad Nacional de Tucumán, República Argentina.
Figura 15: Epidermis abaxial de la hoja de Ficus benjamina, zonas nerval e internerval. $100 \mathrm{X}$

\section{DISCUSIÓN}

De las especies estudiadas, Hedera helix y Vinca difformis presentan células epidérmicas de contorno lobulado, mientras que Evonymus japonicus y Ficus benjamina tienen células poligonales. Esto podría incorporarse en la clave dicotómica como un dilema que separe dos grandes grupos según sus características generales.

Las cuatro especies descriptas presentan estomas sólo en la zona internerval de la cara abaxial de sus hojas. Eso significa que, para realizar una correcta identificación de las mismas, necesitamos contar con fragmentos de ambas epidermis, ya que la epidermis adaxial, excepto la forma de las células, no presenta caracteres diferenciales relevantes.

Se encontraron tricomas simples solamente en la zona nerval de la epidermis adaxial de Vinca difformis, las otras especies estudiadas presentan epidermis completamente glabras.

Los caracteres histológicos encontrados en las especies estudiadas y volcados en las descripciones, constituyen un aporte de gran utilidad para la confección de una clave dicotómica de indentificación de estas plantas tóxicas a través de sus epidermis.

\section{AGRADECIMIENTOS}

A las Srtas. María Mercedes Medina, Lía Inés Coronel y Ana Cecilia Jalil y al Sr. Emanuel Mulet por la confección de los preparados microscópicos.

Al Sr. Gabriel Durango por la
toma de las fotomicrografías.

\section{REFERENCIAS BIBLIOGRÁFICAS}

[1]. Cutler, D. F. Anatomía Vegetal Aplicada. Librería Agropecuaria S. A. 1987

[2]. Baungartner, L. L. and Martin, A. C. Plant histology as aid in squirrel food habits studies. J. Wild. Manage. 3: (1939) 266-268

[3]. Pelliza de Sbriller, A. Acerca de la Microhistología. INTA Comunicación Técnica № 32. Recursos Naturales. Dieta. Estación Experimental Agropecuaria S. C. de Bariloche. 1993.

[4]. Castellaro G, Giorgio, Squella N, Fernando , Ullrich R, Tamara. Algunas Técnicas Microhistolóigcas Utilizadas en la Determinación de la Composición Botánica de Dietas de Herbívoros. Agric. Téc. [online] Vol. 67, № 1, (2007) 86-93. Disponible en la World Wide <http://www.scielo.cl/scielo.php?script=sci_arttext\&pid=S036528072007000100011\&lng=es\&nrm=iso>.

[5]. Catán , A., C. A. M. Degano , L. Larcher . Modificaciones a la técnica microhistológica de Peña Neira para especies forrajeras del Chaco Semiárido Argentino. Quebracho. Revista de Ciencias Forestales (010): [en línea]
(2003)
Disponible
en
la
World
Wide
Web: 
<http://redalyc.uaemex.mx/redalyc/src/inicio/ArtPdfRed.jsp?iCve=48101008>

[6]. Würschmidt, A. E. Y H. Salas. Clave para la determinación de la dieta de herbívoros de alta montaña en Tucumán. Acta Zoológica Lilloana XXXIX:2 (1990) 5-15

[7]. Giorgio Castellaro G., Tamara Ullrich R., Birgit Wackwitz y Alberto Raggi S. Composición botánica de la dieta de alpacas (Lama pacos L.) y llamas (Lama glama L.) en dos estaciones del año, en praderas altiplánicas de un sector de la Provincia de Parinacota, Chile. Agricultura Técnica, Chillán, 64(4). (2004)

[8]. Nasca de Zamora, P. S.; M. B. Colombo; F. G. Raya; J. E. Lucas; M. Lotti de Santos; D. Fernández de Aráoz y G. O. Martín. "Estructura de la epidermis foliar de especies nativas del Chaco Semiárido del Dpto. Río Hondo, Santiago del Estero" Resúmenes de las XII Jornadas Científicas de la Sociedad de Biología de Tucumán, Argentina. (1995)

[9]. Nasca de Zamora, Patricia y Martín, Guillermo. "Clave de Caracteres Epidérmicos Foliares de Malváceas del Chaco Semiárido del Noroeste Argentino" Libro de Resúmenes del VII Congreso Latinoamericano de Botánica y XIV Congreso Mexicano de Botánica. (1998)

[10]. Monteverde Susana. Intoxicaciones causadas por plantas Ornamentales y de Jardín. Asociación Argentina de Medicina Felina. http://www.aamefe.org/intoxicaciones_plantas_monteverde.htm

[11]. Zeinsteger, P.A. y Gurni, A. A Plantas tóxicas que afectan el aparato digestivo de caninos y felinos. Rev. vet. 15:1, 35-44. 2004.

[12]. D’Ambrogio de Argüeso, A. Manual de Técnicas en Histología Vegetal. Editorial Hemisferio Sur S.A. 1986.

[13]. Dizeo de Strittmatter, C. G. Nueva Técnica de Diafanización. Boletín de la Sociedad Argentina de Botánica. Vol. XV, № 1. (1973).126-129

[14]. Van Cotthem, W. R. J. A classfication of stomatal types. Bot. J. Linn. Soc. 63: (1970). 235-246

[15]. Uphof, J. C. T. Plant hairs. En K. Linsbauer, Handbuch der Pflanzenanatomie, Bd 4, T. 5. gebr. Borntraeger, Berlín. 1962.

E-mail: fliazamora@yahoo.com 